\title{
Factors associated with bacteraemia due to multidrug-resistant organisms among bacteraemic patients with multidrug- resistant organism carriage: a case control study
}

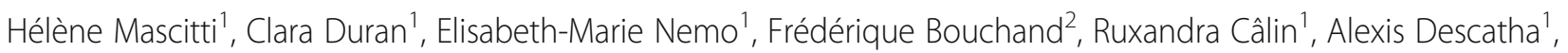
Jean-Louis Gaillard ${ }^{3}$, Christine Lawrence ${ }^{3}$, Benjamin Davido ${ }^{1}$, François Barbier ${ }^{4}$ and Aurélien Dinh ${ }^{1 *}$ (D)

\begin{abstract}
Background: Infections caused by multidrug-resistant organisms (MDRO) are emerging worldwide. Physicians are increasingly faced with the question of whether patients need empiric antibiotic treatment covering these pathogens. This question is especially essential among MDRO carriers. We aim to determine the occurrence of MDRO bacteraemia among bacteraemic patients colonized with MDRO, and the associated factors with MDRO bacteraemia among this population.

Methods: We performed a retrospective monocentric study among MDRO carriers hospitalized with bacteraemia between January 2013 and August 2016 in a French hospital. We compared characteristics of patients with MDRO and non-MDRO bacteraemia.

Results: Overall, 368 episodes of bacteraemia were reviewed; 98/368 (26.6\%) occurred among MDRO carriers. Main colonizing bacteria were extended-spectrum beta-lactamase (ESBL)-producing Escherichia coli (40/98; 40.8\%), ESBL-producing Klebsiella pneumoniae (35/98; 35.7\%); methicillin-resistant Staphylococcus aureus (26/98; 26.5\%) and multidrug-resistant Pseudomonas aeruginosa (PA) (12/98; 12.2\%).

There was no significant difference considering population with MDRO bacteraemia vs. non-MDRO bacteraemia, except for immunosuppression [OR 2.86; $p=0.0207$ ], severity of the episode [OR 3.13; $p=0.0232$ ], carriage of PA [OR 5.24; $p=0.0395]$, and hospital-acquired infection [OR 2.49; $p=0.034]$.

In the multivariate analysis, factors significantly associated with MDRO bacteraemia among colonized patient were only immunosuppression [OR $=2.96 ; p=0.0354]$ and the hospital-acquired origin of bacteraemia $[\mathrm{OR}=2.62 ; p=0.0427]$.

Conclusions: According to our study, occurrence of bacteraemia due to MDRO among MDRO carriers was high. Factors associated with MDRO bacteraemia were severity of the episode and hospital-acquired origin of the bacteraemia. Thus, during bacteraemia among patients colonized with MDRO, if such characteristics are present, broadspectrum antimicrobial treatment is recommended.
\end{abstract}

Keywords: Multidrug-resistant organism, Antimicrobial, Bacteraemia, Carriage

\footnotetext{
* Correspondence: aurelien.dinh@aphp.fr

1 Infectious disease unit, Raymond Poincaré University Hospital, AP-HP,

Versailles Saint-Quentin University, 104 Bd R. Poincaré, 92380 Garches, France

Full list of author information is available at the end of the article
}

(c) The Author(s). 2018 Open Access This article is distributed under the terms of the Creative Commons Attribution 4.0 International License (http://creativecommons.org/licenses/by/4.0/), which permits unrestricted use, distribution, and reproduction in any medium, provided you give appropriate credit to the original author(s) and the source, provide a link to the Creative Commons license, and indicate if changes were made. The Creative Commons Public Domain Dedication waiver (http://creativecommons.org/publicdomain/zero/1.0/) applies to the data made available in this article, unless otherwise stated. 


\section{Background}

There is currently an epidemiologic dramatic increase of multidrug-resistant organisms (MDRO) [1-5].

Infections caused by MDRO have been associated with severe adverse clinical outcomes, leading to increased mortality, prolonged hospital stay, and increased costs, mostly because of delayed effective therapy [6-9]. This dramatic spread takes place in both the community and hospital setting.

However, colonization and infection due to MDRO should be differentiated.

At this time, colonization with MDRO among patients is more frequent than infection.

But colonization with MDRO is a risk factor for infections due to MDRO, especially in transplanted patients and in intensive care unit [10-12].

If sepsis or sepsis-mimicking events occur among MDRO carriers, effective probabilistic broad-spectrum antibiotics are often prescribed in common practice [13]. Consequently, broad-spectrum antimicrobial treatments are increasingly used as empiric therapy among colonized patients. It could lead to unnecessary antibiotic exposure and selective pressure, creating more bacterial resistance.

This vicious circle is worryingly contributing to a rapid international dissemination of MDRO [14-16].

Physicians should therefore consider a prudent use of broad-spectrum antibiotics to limit new emergence of MDRO.

This requires updated studies to identify current risk factors for MDRO infection among MDRO carriers.

The primary objective of our study was to determine the occurrence of MDRO bacteraemia among bacteraemic patients colonized with MDRO, and which associated factors are predictive of bacteraemia due to MDRO among this population.

\section{Methods}

\section{Settings and design}

We performed a retrospective monocentric study among MDRO carriers (from any site: urine, respiratory, digestive, cutaneous), hospitalized with bacteraemia between January 2013 and August 2016 in our teaching hospital, according to STROBE statement [17]. We compared characteristics of patients with MDRO and non-MDRO bacteraemia.

Our university hospital is a disability referral centre for neurological impairment, including spinal cord injured patients. These patients are subject to high antimicrobial exposure because they might have a high rate of infections, especially urinary tract infections; they are also at increased risk of infection with multidrug-resistant bacteria [18-20]. The hospital has 255 acute-care facility beds (including 28 beds of intensive care unit) and 108 for rehabilitation, with around 8400 admissions annually.
Average hospital stays are 6.9 days for acute care and 36.5 days for rehabilitation.

An active surveillance policy for MDRO carriage among high-risk patients is implemented: nasal swab for methicillin-resistant Staphylococcus aureus (MRSA), and rectal swab for Gram negative resistant bacteria and vancomycin-resistant enterococci.

Systematic screening is performed at hospital admission for all patients coming from acute or long-term care facilities, and for community patients previously known as carriers.

Moreover, weekly screening is performed in our intensive care and surgery departments.

All hospitalized patients with positive blood cultures for bacteria were identified from the microbiology laboratory database, and microbiological data was obtained and reviewed. Patients with MDRO carriage (at least one site) during the last 3 months until day of sepsis were included.

Medical charts were reviewed using a standardized data set to collect: demographic characteristics (age, sex, comorbidities, risk factors, etc); clinical, biological, and microbiological data (clinical and severity signs, laboratory tests, organisms identified), and outcomes of each episode.

Blood cultures were performed using aerobic and anaerobic blood culture vials incubated in a Bactec FX instrument (Bactec Ped+ and Lytic/10 Anaerobic/F, BD Diagnostics, Le Pont de Claix, France). The positive blood culture vials were subcultured on blood and chocolate Polyvitex agar plates. All isolates were then identified using MALDI-TOF mass spectrometry (Maldi Biotyper 3.0, Bruker Daltonique, Marnes la Vallée, France).

Antimicrobial susceptibility testing was carried out using the agar disk diffusion method (Bio-Rad) or an automated broth microdilution method (Phoenix, BD Diagnostics, Oxford, UK). The breakpoints used were those defined by the French Committee for Antimicrobial Susceptibility Testing (http://www.sfm-microbiologie.org/UserFiles/files/ casfm/CASFM\%20V1_0\%20FEV_2018.pdf).

\section{Definitions}

Bacteraemia was defined as the association of at least one positive blood culture and a prescription of a systemic antibiotic treatment to treat bacteraemia. For common skin contaminants, such as coagulase-negative staphylococci or Corynebacterium sp., at least two different sets of blood cultures were required.

Polymicrobial bacteraemia was defined as having more than one organism found in the same bacteraemic episode.

MDRO status was determined for the Enterobacteriaceae group, Acinetobacter sp., Pseudomonas aeruginosa, and Enterococcus sp. as acquired non-susceptibility to at 
least one agent in three or more antimicrobial categories; for Staphylococcus aureus as resistance to methicillin [21].

High zone of prevalence of MDRO were southern Europe (Spain, Italy, Greece), North Africa and Asia according to European Centre for Disease Prevention and Control (ECDC) data (https://www.ecdc.europa.eu/en/ home).

Hospital-acquired infection was determined as clinical signs of infection or infection arise at least $48 \mathrm{~h}$ after hospital admission.

Prior colonization was defined as isolation of MDRO from any site without any clinical signs of inflammation or sepsis, and antibiotic therapies targeting these MDRO, within a designated period of 3 months before the day of bacteraemia.

Prior antibiotic use was defined as the use of at least 1 dose of any antimicrobial treatment in a designated period of 3 months until the day before sepsis.

Immunosuppression included the following: diabetes mellitus, ongoing neoplasia, hemopathy, HIV, hypogamma globulinemia, immunosuppressive therapy (ie. corticotherapy $>20 \mathrm{mg} / \mathrm{d}$, chemotherapy or immunosuppressive treatment such as cyclophosphamide, azathioprine and cyclosporine).

Primary site of infection were clinically suspected (by the physician in charge or reported on the medical chart) or bacteriologically documented with the same bacterial identification as that in the blood culture. Primary sites were categorized as urinary tract infection, catheter line-associated bacteraemia, osteoarticular infection, pulmonary tract infection, skin and soft tissue infection, intra-abdominal infection, and unknown when no primary site had been identified.

Severity was defined as the requirement of at least one of the following criteria: volume expansion, assisted (mechanical) ventilation, vasopressor requirement, and intensive care unit (ICU) admission during the episode.

Cure was defined as the absence of clinical and biological signs of infection at 1 month after end of antimicrobial treatment or at hospital discharge without any additional antimicrobial treatment.

Mortality was defined as dead status before 30 days after the end of antimicrobial treatment.

\section{Statistical analysis}

All continuous variables are presented as mean and standard deviation, and the categorical variables are presented as frequencies. Correlations between risk factors and MDRO bacteraemia among patients colonized with MDRO were determined by Student's t-test for continuous variables and the Pearson's $\chi^{2}$ test for categorical variables.

Univariate analysis and multivariate analysis were performed. Variable for multivariate analysis were all associated risks that had a $p$-value $\leq 0.05$ and sex in the univariate analysis.

The relative risks of MDROs bacteraemia were estimated by calculating the adjusted odds ratios (OR) and corresponding 95\% confidence intervals (CI).

All reported probability values ( $P$-values) were based on two-sided tests, and a $P$-value of 0.05 was considered statistically significant. All analyses were performed using the Statistical Package for Social Science (SPSS) version 17.0 (SPSS, Chicago, IL, USA).

\section{Results}

During the study period, a mean of $198 \pm 54$ screening per month was performed, and mean positive results for MDRO per patient was $23 \pm 5 \%$, with $45 \%$ of extended-spectrum beta-lactamase (ESBL)-producers isolates.

In total, 368 episodes of bacteraemia were reviewed; 98 (26.6\%) occurred among 77 MDRO carriers (Fig. 1). Eight bacteraemia episodes were plurimicrobial.

Considering the 98 episodes of bacteraemia among MDRO carriers, mean age was 55.8 years old, and sex ratio was 1.65. Prior antimicrobial treatment in the last 3 months occurred in $66(67.3 \%)$ cases, 42 (42.9\%) patients had an indwelling catheter, 33 (33.7\%) were immunosuppressed, and 55 (56.1\%) were considered as severe.

Main primary site of infections were urinary tract infections $(25 ; 25.5 \%)$ and catheter-line associated infections $(25 ; 25.5 \%) ; 12$ (12.2\%) patients presented primary bacteraemia.

Bacteraemia were hospital-acquired in 62 (63.3\%) cases.

The rate of bacteraemia due to MDRO was $53.1 \%$ $(n=52)$ (Table 1). Among them, 41 (78.8\%) episodes were due to multidrug-resistant Enterobacteriaceae, of which 22 (42.3\%) were due to ESBL Enterobacteriaceae.

Overall, main colonizing bacteria were ESBL-producing Escherichia coli (EC) ( $n=40 ; 40.8 \%)$, ESBL-producing Klebsiella pneumoniae (KP) $(n=35 ; 35.7 \%)$; MRSA $(n=26$; $26.5 \%)$, and Pseudomonas aeruginosa (PA) $(n=12 ; 12.2 \%)$. Twenty-five patients (for 32 episodes) were carriers of several MDRO. Sites of carriage and microorganisms identified are presented in Table 2.

Among carriers with bacteraemia due to MDRO, a discordant identification between carriage and bacteraemia was found in 23 (44.2\%) episodes (Table 3).

On the contrary, 29 (55.8\%) episodes had a concordant identification, which were due to ESBL KP $(n=10)$, ESBL EC $(n=7)$, MRSA $(n=4)$, VIM-type carbapenemase-producing PA $(\mathrm{n}=3), \mathrm{ESBL}$ Enterobacter cloacae $(n=2)$, ceftaroline-resistant PA $(n=1)$, carbapenemase-producing E. cloacae $(n=1)$, and ceftaroline-resistant Acinetobacter baumanii $(n=1)$. Sites of carriage were rectal $(n=18)$, urinary $(n=14)$, respiratory $(n=11)$ and cutaneous $(n=7)$.

The global cure rate was $83 / 98$ (84.6\%). 


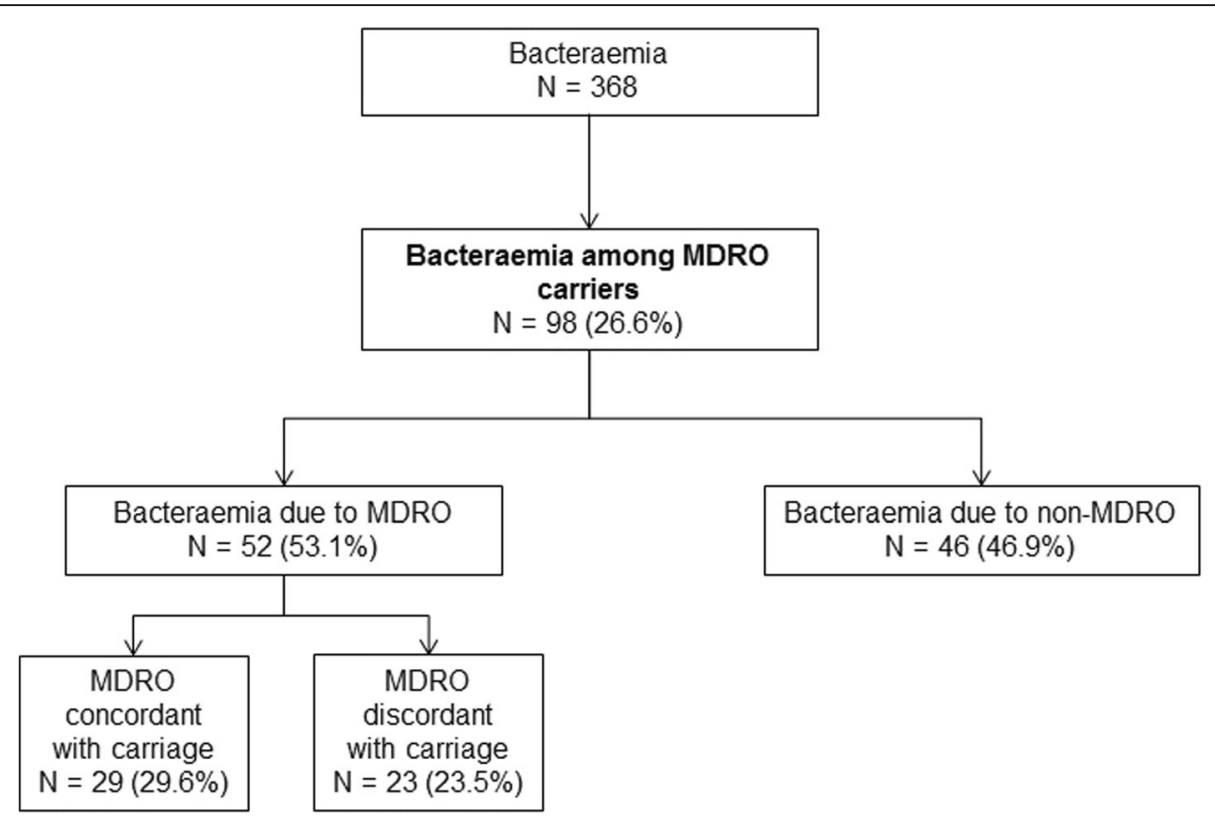

Fig. 1 Study flow chart

In univariate analysis, there was no significant difference considering population with MDRO bacteraemia vs. non-MDRO bacteraemia, except for immunosuppression [OR 2.86; $p=0.0207$ ], severity of the episode [OR 3.13; $p=0.0232$ ], carriage of Pseudomonas aeruginosa [OR 5.24; $p=0.0395]$, and hospital-acquired infection [OR 2.49; $p=0.034$ ] (Table 4).

In the multivariate analysis (Table 4), factors significantly associated with MDRO bacteraemia among colonized patient were only immunosuppression $[\mathrm{OR}=2.96$; $p=0.0354]$, and the nosocomial origin of bacteraemia [OR $=2.62 ; p=0.0427$.

\section{Discussion}

In our study, the rate of MDRO bacteraemia among bacteraemic patients colonized with MDRO is high (53.1\%).

Main factors associated with MDRO bacteraemia in those patients are immunosuppression, severity of the episode, colonization with Pseudomonas aeruginosa, and nosocomial infection in univariate analysis. In multivariate analysis, the only significant factors found are severity of the episode and the nosocomial origin of the infection.

The originality of our study is to focus on bacteraemic patient colonized with MDRO. Our main question is: when should we treat with probabilistic broad-spectrum antimicrobial treatment patients with known MDRO colonization and positive blood cultures?

\section{Risk factor for MDRO/ESBL infections}

Most studies focused on colonization and infections due to multidrug-resistant Enterobacteriaceae, in ICU, or among immunosuppressed patients.
For example, in a 6-year prospective study, Razazi et al. screened 6303 patients admitted in ICU [22]; 843 (13.4\%) had ESBL Enterobacteriaceae carriage detected. Among those carriers, 111 (13\%) patients developed ICU-acquired pneumonia, of whom only 48 (43\%) had ESBL Enterobacteriaceae pneumonia (6\% of carriers). Moreover, considering ventilator-acquired pneumonia in ICU patients, Bruyère et al. noted in their retrospective study that the positive predictive value of digestive ESBL Enterobacteriaceae colonization for ESBL Enterobacteriaceae pneumonia was also low (41.5\%) [23].

More generally, in a prospective multicenter cohort study in ICU, Barbier et al. demonstrated that ESBL Enterobacteriaceae infections increased carbapenem consumption, length of stay and day 28 mortality [24]. Also, ESBL Enterobacteriaceae infections (16.4\%) were rather infrequent in carriers.

In Holland, a study focused on the predictive value of prior colonization for third-generation cephalosporin-resistant Enterobacteriaceae for infection due to the same microorganism [25]. This study was performed in all medical wards of an hospital, ICU included. The authors noted that, among 9422 episodes, 1657 (17.6\%) of colonized patients were bacteraemic, and 64 (3.8\%) were colonized with third-generation cephalosporin-resistant Enterobacteriaceae.

In this study, the occurrence of MDRO bacteraemia was low, corresponding to usual epidemiological data in Holland. In our study, the rate of infection due to MDRO is higher which may be due to local epidemiology.

Finally, an Israelian cohort study, with 431 carriers of carbapenem-resistant Klebsiella pneumonia (CRKP) 
Table 1 Main characteristics of multidrug-resistant organism carriers with bacteraemia

\begin{tabular}{|c|c|c|c|c|}
\hline Variable & $\begin{array}{l}\text { Non MDR bacteraemia } \\
(n=46)\end{array}$ & $\begin{array}{l}\text { MDR bacteraemia } \\
(n=52)\end{array}$ & Odds Ratio & $P$ value \\
\hline Sex (male) & $30(65.2 \%)$ & $31(59.6 \%)$ & $0.70[0.35 ; 1.79]$ & 0.5683 \\
\hline Recent ( $<3$ months) trip in zone with high MDRO prevalence ${ }^{a}$ & $6(13.0 \%)$ & $3(5.8 \%)$ & $0.41[0.10 ; 1.74]$ & 0.2250 \\
\hline Prior antimicrobial treatment in last 6 months & $28(60.9 \%)$ & $38(73.1 \%)$ & $1.74[0.74 ; 4.09]$ & 0.2004 \\
\hline Urinary indwelling catheter & $19(41.3 \%)$ & $23(44.2 \%)$ & $1.13[0.51 ; 2.51]$ & 0.7704 \\
\hline Immunosuppression & $10(21.7 \%)$ & $23(44.2 \%)$ & $2.86[1.17 ; 6.95]$ & 0.0207 \\
\hline Severity & $7(15.2 \%)$ & $19(36.5 \%)$ & $3.13[1.17 ; 8.36]$ & 0.0232 \\
\hline \multicolumn{5}{|l|}{ Primary site of infection } \\
\hline UTI & $10(21.7 \%)$ & $15(28.8 \%)$ & $1.46[0.58 ; 3.67]$ & 0.4218 \\
\hline Intra abdominal infection & $6(13.0 \%)$ & $5(9.6 \%)$ & $0.71[0.20 ; 2.50]$ & 0.5929 \\
\hline Bone and joint infection & $4(8.7 \%)$ & $0(0.0 \%)$ & $0.00[0.00 ; 1]$ & 0.9710 \\
\hline Respiratory tract infection & $2(4.3 \%)$ & $6(11.5 \%)$ & $2.87[0.55 ; 14.98]$ & 0.2113 \\
\hline Skin soft tissue infection & $7(15.2 \%)$ & $4(7.7 \%)$ & $0.46[0.13 ; 1.70]$ & 0.2471 \\
\hline Catheter line associated infcetion & $10(21.7 \%)$ & $15(28.8 \%)$ & $1.46[0.58 ; 3.67]$ & 0.4218 \\
\hline No primary site of infection & $5(10.9 \%)$ & $7(13.5 \%)$ & $1.28[0.38 ; 4.33]$ & 0.6965 \\
\hline \multicolumn{5}{|l|}{ Colonization MDR pathogen } \\
\hline Polymicrobial & $15(32.6 \%)$ & $17(32.7 \%)$ & & \\
\hline CRE & $1(2.2 \%)$ & $2(3.8 \%)$ & & \\
\hline ESBL Escherichia coli & $23(50.0 \%)$ & $17(32.7 \%)$ & $0.49[0.22 ; 1.09]$ & 0.0814 \\
\hline Klebsiella spp. & $18(39.1 \%)$ & $25(48.1 \%)$ & $1.44[0.64 ; 3.22]$ & 0.3738 \\
\hline ESBL Klebsiella spp. & $17(37.0 \%)$ & $23(44.2 \%)$ & & \\
\hline Carba-R Klebsiella spp. & $1(2.2 \%)$ & $1(1.9 \%)$ & & \\
\hline CASE Klebsiella spp. & $0(0.0 \%)$ & $1(1.9 \%)$ & & \\
\hline Citrobacter spp. & $1(2.2 \%)$ & $2(3.8 \%)$ & $1.80[0.16 ; 20.53]$ & 0.6360 \\
\hline ESBL Citrobacter spp. & $1(2.2 \%)$ & $2(3.8 \%)$ & & \\
\hline Enterobacter spp. & $5(10.9 \%)$ & $8(15.4 \%)$ & $1.63[0.45 ; 5.98]$ & 0.4590 \\
\hline ESBL Enterobacter spp. & $5(10.9 \%)$ & $7(13.5 \%)$ & & \\
\hline Carba-R Enterobacter spp. & $0(0.0 \%)$ & $1(1.9 \%)$ & & \\
\hline Acinetobacter baumanii & $2(4.3 \%)$ & $3(5.8 \%)$ & $0.43[0.04 ; 4.92]$ & 0.4984 \\
\hline ESBL A. baumanii & $1(2.2 \%)$ & $1(1.9 \%)$ & & \\
\hline Carba-R A. baumanii & $1(2.2 \%)$ & $1(1.9 \%)$ & & \\
\hline Cefta-R A. baumanii & $0(0.0 \%)$ & $1(1.9 \%)$ & & \\
\hline Pseudomonas aeruginosa & $2(4.3 \%)$ & $10(19.2 \%)$ & $5.24[1.08 ; 25.32]$ & 0.0395 \\
\hline ESBL $P$. aeruginosa & $0(0.0 \%)$ & $1(1.9 \%)$ & & \\
\hline Carba-R P. aeruginosa & $0(0.0 \%)$ & $3(5.8 \%)$ & & \\
\hline Cefta-R P. aeruginosa & $2(4.3 \%)$ & $6(11.5 \%)$ & & \\
\hline MRSA & $11(23.9 \%)$ & $15(28.8 \%)$ & $1.29[0.52 ; 3.19]$ & 0.5814 \\
\hline VRE & $1(2.2 \%)$ & $0(0.0 \%)$ & $0.88[0.05 ; 14.51]$ & 0.9300 \\
\hline \multicolumn{5}{|l|}{ Type of infections } \\
\hline Nosocomial & $24(52.2 \%)$ & $38(73.1 \%)$ & $2.49[1.07 ; 5.78]$ & 0.0340 \\
\hline Cure rate & $39(84.8 \%)$ & $44(84.6 \%)$ & $0.99[0.33 ; 2.97]$ & 0.9817 \\
\hline
\end{tabular}

${ }^{a}$ Geographic area with high incidence of extended-spectrum beta-lactamase-producing bacteria, CRE and VRE: Southern Europe (Spain, Italy, Greece), North Africa and Asia

Carba- $R$ Carbapenem-resistant; CASE Cephalosporinase-producing; Cefta- $R$ Ceftaroline-resistant; CRE Carbapenem-resistant Enterobacteriaceae;

ESBL Extended-spectrum beta-lactamase; MDR Multidrug-resistant; MRSA Methicillin-resistant Staphylococcus aureus; VRE Vancomycin-resistant Enterococci

italicised valued are statistically significant 
Table 2 Multidrug-resistant organism carriage according to site

\begin{tabular}{lllll}
\hline & Urinary & Rectal & Respiratory & Cutaneous / Wound \\
\hline ESBL Enterobacteriaceae & 30 & 59 & 2 & 13 \\
CRE (NDM + OXA types) & 1 & 2 & 0 & 0 \\
CASE Enterobacteriaceae & 0 & 1 & 0 & 0 \\
ESBL Pseudomonas aeruginosa & 1 & 0 & 0 & 3 \\
Carba-R P. aeruginosa & 0 & 2 & 0 & 2 \\
Cefta-R P. aeruginosa & 0 & 2 & 3 & 0 \\
ESBL Acinetobacter baumanii & 0 & 1 & 0 & 0 \\
OXA-23 A. baumanii & 0 & 2 & 0 & 0 \\
Cefta-R A. baumanii & 0 & 1 & 0 & 0 \\
MRSA & 3 & 0 & 19 & 0 \\
VRE & 0 & 1 & 0 & 0
\end{tabular}

ESBL Extended-spectrum beta-lactamase; CRE Carbapenem-resistant Enterobacteriaceae; CASE Cephalosporinase; Carba- $R$ Carbapenem-resistant; Cefta- $R$ Ceftaroline-resistant; MRSA Methicillin-resistant Staphylococcus aureus; VRE Vancomycin-resistant Enterococci

Table 3 Discordant identification between carriage and blood culture

\begin{tabular}{|c|c|}
\hline Carriage MDRO & Blood culture MDRO \\
\hline ESBL Escherichia coli & MDR non-ESBL E. coli \\
\hline ESBL E. coli & ESBL K. pneumoniae \\
\hline ESBL E. coli & ESBL K. pneumoniae \\
\hline CASE Klebsiella pneumoniae & MDR K. pneumoniae \\
\hline ESBL K. pneumoniae & MDR non-ESBL E. coli \\
\hline ESBL K. pneumoniae & MDR K. pneumoniae \\
\hline ESBL K. pneumoniae & MDR Proteus mirabilis \\
\hline ESBL K. pneumoniae & MDR Serratia marcescens \\
\hline ESBL K. pneumoniae & Cefta-R P. aeruginosa \\
\hline Cefta-R Pseudomonas aeruginosa & MDR non-ESBL E. coli \\
\hline Cefta-R P. aeruginosa & MDR Enterobacter cloacae \\
\hline ESBL E. cloacae & MDR P. mirabilis \\
\hline ESBL Acinetobacter baumanii & MDR non-ESBL E. coli \\
\hline Cefta-R A. baumanii & MDR $P$. aeruginosa \\
\hline ESBL Morganella morganii & MDR Providencia stuartii \\
\hline MRSA & MDR non-ESBL E. coli \\
\hline MRSA & MDR non-ESBL E. coli \\
\hline MRSA & ESBL K. pneumoniae \\
\hline MRSA & MDR Enterococcus faecium \\
\hline ESBL E. coliESBL K. pneumoniaeMRSA & MDR non-ESBL E. coli \\
\hline ESBL E. coliESBL K. oxytoca ESBL Citrobacter sp.ESBL M. morganii MRSA & MDR P. mirabilis \\
\hline ESBL E. coliESBL K. pneumoniae & MDR non-ESBL E. coli \\
\hline ESBL E. coliMRSA & MDR non-ESBL E. coli \\
\hline
\end{tabular}

CASE Cephalosporinase-producing; Cefta- $R$ Ceftaroline-resistant; ESBL Extended-spectrum beta-lactamase; MDRO Multidrug-resistant organism; MRSA Methicillin-resistant Staphylococcus aureus 
Table 4 Multivariate analysis associated with multidrug-resistant organism bacteraemia

\begin{tabular}{|c|c|c|c|c|c|}
\hline \multirow[t]{2}{*}{ Variable } & \multirow{2}{*}{$\begin{array}{l}\text { MDR } \\
\text { bacteraemia }\end{array}$} & \multicolumn{2}{|l|}{ Univariate analysis } & \multicolumn{2}{|c|}{ Multivariate analysis } \\
\hline & & Odds Ratio & $P$ value & Odds Ratio & $P$ value \\
\hline Sex (male) & $31 / 61(50.8 \%)$ & $0.70[0.35 ; 1.79]$ & 0.5683 & $1.04[0.40 ; 2.70]$ & 0.9403 \\
\hline Immunosuppression & 23/33 (69.7\%) & $2.86[1.17 ; 6.95]$ & 0.0207 & $2.96[1.08 ; 8.13]$ & 0.0354 \\
\hline Severity & 19/26 (73.1\%) & $3.13[1.17 ; 8.36]$ & 0.0232 & $2.32[0.78 ; 6.88]$ & 0.1303 \\
\hline Colonization MDR Pseudomonas aeruginosa & 10/12 (83.3\%) & $5.24[1.08 ; 25.32]$ & 0.0395 & $2.95[0.49 ; 17.77]$ & 0.2386 \\
\hline Hospital-acquired & $38 / 62$ (61.3\%) & $2.49[1.07 ; 5.78]$ & 0.034 & $2.62[1.03 ; 6.64]$ & 0.0427 \\
\hline
\end{tabular}

MDR Multidrug-resistant

italicised valued are statistically significant

included, noted that the rate of bloodstream infections (BSI) was $20 \%$ and rate of BSI due to Gram negative resistant bacteria was $80 \%(68 / 85)$ [26]. Among them, 19 BSI were due to CRKP and 20 to ESBL Enterobacteriaceae. However, in this study, no prognostic factors of CRKP BSI were identified.

The authors concluded that this raises the question regarding the use of probabilistic broad-spectrum antibiotic therapy for MDRO carriers who develop severe sepsis, as in our study.

Moreover, the authors also described frequent discordance between bacteria involved in carriage and in blood cultures [26].

Carriage of MDRO is generally the marker of high antibiotic exposure of the patient, which induces selective pressure on all flora. Yet, all MDRO are not screened, and usual screening techniques are not $100 \%$ sensitive. Therefore, a MDRO not identified during screening could be responsible for sepsis. But the indication of broad-spectrum antimicrobial treatment during sepsis among patients with MDRO carriage is still under debate, as patients do have a higher risk of MDRO infection, even if due to a different microorganism.

\section{Risks associated with infection due to MDRO}

Regarding infection due to MRSA, colonization by MRSA is a well-known risk factor [27-29], especially in critically ill neonates children [30].

Thus, risk factors for infection due to MDRO is a complex phenomenon due to various microbiological, clinical, demographic and anamnestic characteristics [22, 23, 31, 32].

Use of algorithm to limit unnecessary use of broad-spectrum antimicrobial treatment should be encouraged [31, 32], as the one suggested by $M$. Basseti and J. Rodriguez Baño, which includes simple and easy to collect criteria: severity of the episode, community-acquired character, previous colonization to MDRO, indwelling device, age and previous exposure to antibiotic [33].

Lastly, new rapid diagnosis tests for bacterial resistance could help to avoid unnecessary broad-spectrum antimicrobial treatment among bacteraemic population known to be colonized by MDRO [34-37].

\section{Bias and weakness}

The bias and weakness of our study are due to its monocentric and retrospective design, and limited sample size. Some data may be missing such as previous antimicrobial prescriptions due to memory bias. All patients were not systematically screened for MDRO at every site. Still, we studied several MDRO (ESBL bacteria, carbapenem-resistant Enterobacteriaceae and MRSA for example) and different sites of carriage which reflect every day practice in a tertiary care hospital. Finally, another limit of this work is that we were not able to identify patients with re-hospitalization or transferred from another hospital, which could imply an underestimation of the proportion of hospital-acquired infections.

Future research is needed to better understand the link between colonization and infection due to MDRO.

\section{Conclusions}

According to our study, occurrence of bacteraemia due to MDRO among bacteraemic MDRO carriers was high. However, concordance between carried bacteria and blood culture bacteria was not always consistent.

Factors associated with MDRO bacteraemia were severity of the episode and nosocomial origin of the bacteraemia.

Thus, during bacteraemia among patients colonized with MDRO, if characteristics above described are present, broad-spectrum antimicrobial treatment is recommended.

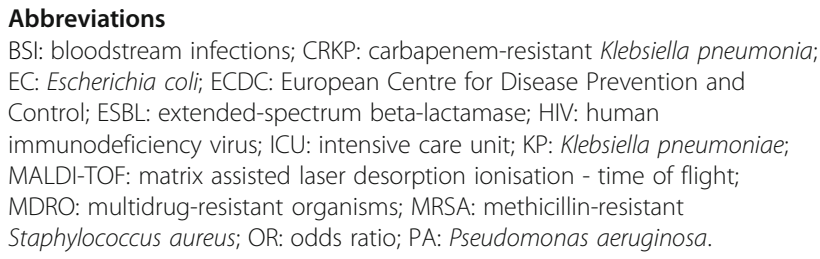

\section{Acknowledgments}

The authors would like to thank Elodie Choisy from the infectious disease unit of Raymond Poincaré Hospital for her help and support.

\section{Availability of data and materials}

Data upon which this paper is based are available from the primary author upon request.

\section{Authors' contributions}

ADi developed the study design. JLG and CL performed all laboratory tests. $\mathrm{HM}, \mathrm{CD}$ and EMN were responsible for data collection. ADe performed 
statistical analysis. ADi and FBa were responsible for data analysis and data interpretation. CD, FBo, FBa and ADi drafted the first version of the manuscript. All authors revised and approved the final manuscript.

\section{Ethics approval and consent to participate}

As it is a retrospective study, formal consent or approval is not required. Data collected were treated confidentially according to Commission Nationale de l'Informatique et Liberté (CNIL; National Commission of Informatics and Freedom) statements.

\section{Consent for publication}

Not applicable.

\section{Competing interests}

The authors declare that they have no competing interests.

\section{Publisher's Note}

Springer Nature remains neutral with regard to jurisdictional claims in published maps and institutional affiliations.

\section{Author details}

'Infectious disease unit, Raymond Poincaré University Hospital, AP-HP, Versailles Saint-Quentin University, 104 Bd R. Poincaré, 92380 Garches, France. ${ }^{2}$ Pharmacy department, Raymond Poincaré University Hospital, AP-HP, Versailles Saint-Quentin University, 104 Bd R. Poincaré, 92380 Garches, France. ${ }^{3}$ Microbiological laboratory, Raymond Poincaré University Hospital, AP-HP, Versailles Saint-Quentin University, 104 Bd R. Poincaré, 92380 Garches, France. ${ }^{4}$ Intensive care unit, Orléans Hospital, 14 Avenue de l'Hôpital, 45067 Orléans, France.

\section{Received: 22 August 2018 Accepted: 19 September 2018}

Published online: 29 September 2018

\section{References}

1. WHO. Antimicrobial resistance: global report on surveillance 2014 [Internet]. 2014 [cited 2018 Aug 21]. Available from: http://apps.who.int/iris/bitstream/ handle/10665/112642/9789241564748_eng.pdf;jsessionid= 99C1FBE19E0C74F2E8288949002DFE34? sequence $=1$

2. Drieux L, Brossier F, Duquesnoy O, Aubry A, Robert J, Sougakoff W, et al. Increase in hospital-acquired bloodstream infections caused by extended spectrum $\beta$-lactamase-producing Escherichia coli in a large French teaching hospital. Eur J Clin Microbiol Infect Dis. 2009;28:491-8.

3. Saurina G, Quale JM, Manikal VM, Oydna E, Landman D. Antimicrobial resistance in Enterobacteriaceae in Brooklyn, NY: epidemiology and relation to antibiotic usage patterns. J Antimicrob Chemother. 2000;45:895-8.

4. European Centre for Disease Prevention and Control (ECDC). Antimicrobial resistance surveillance in Europe 2016. Annual report of the European Antimicrobial Resistance Surveillance Network (EARS-Net) [Internet]. 2016 [cited 2018 Mar 26]. Available from: https://ecdc.europa.eu/sites/portal/files/ documents/AMR-surveillance-Europe-2016.pdf

5. Centers for Disease Control and Prevention (CDC). Vital Signs: CarbapenemResistant Enterobacteriaceae. Morb Mortal Wkly Rep [Internet]. 2013 [cited 2018 Apr 2];62:165-70. Available from: https://www.cdc.gov/mmwr/preview/ mmwrhtml/mm6209a3.htm

6. Lee SY, Kotapati S, Kuti JL, Nightingale CH, Nicolau DP. Impact of extendedSpectrum $\beta$-lactamase-producing Escherichia coli and Klebsiella species on clinical outcomes and hospital costs: a matched cohort study. Infect Control Hosp Epidemiol. 2006;27:1226-32.

7. Lautenbach E, Patel JB, Bilker WB, Edelstein PH, Fishman NO. ExtendedSpectrum -lactamase-producing Escherichia coli and Klebsiella pneumoniae: risk factors for infection and impact of resistance on outcomes. Clin Infect Dis. 2001;32:1162-71.

8. Blot S, Depuydt P, Vogelaers D, Decruyenaere J, De WJ, Hoste E, et al. Colonization status and appropriate antibiotic therapy for nosocomial bacteremia caused by antibiotic-resistant gram-negative bacteria in an intensive care unit. Infect Control Hosp Epidemiol. 2005;26:575-9.

9. de Kraker MEA, Wolkewitz M, Davey PG, Koller W, Berger J, Nagler J, et al. Burden of antimicrobial resistance in European hospitals: excess mortality and length of hospital stay associated with bloodstream infections due to Escherichia coli resistant to third-generation cephalosporins. J Antimicrob Chemother. 2011;66:398-407.
10. Bonten MJ, Weinstein RA. The role of colonization in the pathogenesis of nosocomial infections. Infect Control Hosp Epidemiol. 1996;17:193-200.

11. Detsis M, Karanika S, Mylonakis E. ICU acquisition rate, risk factors, and clinical significance of digestive tract colonization with extended-Spectrum Beta-lactamase-producing Enterobacteriaceae: a systematic review and meta-analysis. Crit Care Med. 2017;45:705-14.

12. Gómez-Zorrilla S, Camoez M, Tubau F, Cañizares R, Periche E, Dominguez $M A$, et al. Prospective observational study of prior rectal colonization status as a predictor for subsequent development of Pseudomonas aeruginosa clinical infections. Antimicrob Agents Chemother. 2015;59:5213-9.

13. Barbier F, Bailly S, Schwebel C, Papazian L, Azoulay É, Kallel H, et al. Infection-related ventilator-associated complications in ICU patients colonised with extended-spectrum $\beta$-lactamase-producing Enterobacteriaceae. Intensive Care Med. 2018;44:616-26.

14. Karah N, Haldorsen B, Hermansen NO, Tveten $\mathrm{Y}$, Ragnhildstveit E, Skutlaberg $\mathrm{DH}$, et al. Emergence of OXA-carbapenemase- and $16 \mathrm{~S}$ rRNA methylaseproducing international clones of Acinetobacter baumannii in Norway. J Med Microbiol. 2011;60:515-21.

15. Chen $\mathrm{S}, \mathrm{Hu} F, X_{u} \mathrm{X}, \mathrm{Liu}$ Y, Wu W, Zhu D, et al. High prevalence of KPC-2type carbapenemase coupled with CTX-M-type extended-spectrum betalactamases in carbapenem-resistant Klebsiella pneumoniae in a teaching hospital in China. Antimicrob agents Chemother. American Society for Microbiology (ASM). 2011;55:2493-4.

16. Munoz-Price LS, Hayden MK, Lolans K, Won S, Calvert K, Lin M, et al. Successful control of an outbreak of Klebsiella pneumoniae Carbapenemase-Producing K. pneumoniae at a long-term acute care hospital. Infect Control Hosp Epidemiol. 2010;31:341-7.

17. STROBE Statement [Internet]. [cited 2018 Sep 18]. Available from: https://www.strobe-statement.org/index.php?id=strobe-home

18. Dinh A, Saliba M, Saadeh D, Bouchand F, Descatha A, Roux ALL, et al. Blood stream infections due to multidrug-resistant organisms among spinal cordinjured patients, epidemiology over 16 years and associated risks: a comparative study. Spinal Cord [Internet]. 2016 [cited 2017 Mar 11];54:720725. Available from: http://www.nature.com/doifinder/10.1038/sc.2015.234

19. Esposito S, Leone S, Noviello S, Lanniello F, Fiore M. Antibiotic resistance in long-term care facilities. New Microbiol. 2007:30:326-31.

20. Couderc C, Jolivet S, Thiébaut ACM, Ligier C, Remy L, Alvarez A-S, et al. Fluoroquinolone use is a risk factor for methicillin-resistant Staphylococcus aureus Acquisition in Long-term Care Facilities: a nested case-case-control study. Clin Infect Dis. 2014;59:206-15.

21. Magiorakos A-P, Srinivasan A, Carey RB, Carmeli Y, Falagas ME, Giske CG, et al. Multidrug-resistant, extensively drug-resistant and pandrug-resistant bacteria: an international expert proposal for interim standard definitions for acquired resistance. Clin Microbiol Infect. 2012;18:268-81.

22. Razazi K, Mekontso Dessap A, Carteaux G, Jansen C, Decousser J-W, de Prost $\mathrm{N}$, et al. Frequency, associated factors and outcome of multi-drug-resistant intensive care unit-acquired pneumonia among patients colonized with extended-spectrum $\beta$-lactamase-producing Enterobacteriaceae. Ann Intensive Care. 2017;7:61.

23. Bruyère $R$, Vigneron $C$, Bador J, Aho $S$, Toitot $A$, Quenot J, et al. Significance of prior digestive colonization with extended-Spectrum $\beta$-lactamaseproducing Enterobacteriaceae in patients with ventilator-associated pneumonia. Crit care med. Crit Care Med. 2016:44:699-706.

24. Barbier F, Pommier C, Essaied W, Garrouste-Orgeas M, Schwebel C, Ruckly $\mathrm{S}$, et al. Colonization and infection with extended-spectrum $\beta$-lactamaseproducing Enterobacteriaceae in ICU patients: what impact on outcomes and carbapenem exposure? J Antimicrob Chemother. 2016;71:1088-97.

25. Rottier WC, Bamberg YRP, Dorigo-Zetsma JW, van der Linden PD, Ammerlaan HSM, Bonten MJM. Predictive value of prior colonization and antibiotic use for third-generation cephalosporin-resistant Enterobacteriaceae bacteremia in patients with sepsis. Clin Infect Dis. 2015:60:1622-30.

26. Amit S, Mishali H, Kotlovsky T, Schwaber MJ, Carmeli Y. Bloodstream infections among carriers of carbapenem-resistant Klebsiella pneumoniae: etiology, incidence and predictors. Clin Microbiol Infect. 2015:21:30-4.

27. Simor AE, Loeb M, CIDS/CAMM guidelines committee. The management of infection and colonization due to methicillin-resistant Staphylococcus aureus: A CIDS/CAMM position paper. Can J Infect Dis. 2004;15:39-48.

28. Stenehjem E, Rimland D. MRSA nasal colonization burden and risk of MRSA infection. Am J Infect Control. 2013;41:405-10. 
29. Garrouste-Orgeas M, Timsit J-F, Kallel H, Ben AA, Dumay MF, Paoli B, et al. Colonization with methicillin-resistant Staphylococcus aureus in ICU patients morbidity, mortality, and Glycopeptide use. Infect Control Hosp Epidemiol. 2001:22:687-92.

30. Milstone AM, Goldner BW, Ross T, Shepard JW, Carroll KC, Perl TM. Methicillin-resistant Staphylococcus aureus colonization and risk of subsequent infection in critically ill children: importance of preventing nosocomial methicillin-resistant Staphylococcus aureus transmission. Clin Infect Dis. 2011;53:853-9.

31. Tumbarello M, Trecarichi EM, Bassetti M, De Rosa FG, Spanu T, Di Meco E, et al. Identifying patients harboring extended-Spectrum- $\beta$-lactamase-producing Enterobacteriaceae on hospital admission: derivation and validation of a scoring system. Antimicrob Agents Chemother. 2011;55:3485-90.

32. Goodman KE, Lessler J, Cosgrove SE, Harris AD, Lautenbach E, Han JH, et al. A clinical decision tree to predict whether a Bacteremic patient is infected with an extended-Spectrum $\beta$-lactamase-producing organism. Clin Infect Dis. 2016;63:896-903.

33. Bassetti M, Baño JR. Should we take into account ESBLs in empirical antibiotic treatment? Intensive Care Med. 2016;42:2059-2062.

34. Renvoisé A, Decré D, Amarsy-Guerle R, Huang T-D, Jost C, Podglajen I, et al. Evaluation of the $\beta$ Lacta test, a rapid test detecting resistance to thirdgeneration cephalosporins in clinical strains of Enterobacteriaceae. J Clin Microbiol. American Society for Microbiology (ASM). 2013;51:4012-7.

35. Gallah S, Decré $D$, Genel N, Arlet $G$. The $\beta$-Lacta test for direct detection of extended-spectrum- $\beta$-lactamase-producing Enterobacteriaceae in urine. J Clin Microbiol American Society for Microbiology (ASM). 2014:52:3792-4.

36. Delport JA, Strikwerda A, Armstrong A, Schaus D, John M. MALDI-ToF short incubation identification from blood cultures is associated with reduced length of hospitalization and a decrease in bacteremia associated mortality. Eur J Clin Microbiol Infect Dis. 2017;36:1181-6.

37. Beganovic M, Costello M, Wieczorkiewicz SM. Effect of Matrix-Assisted Laser Desorption lonization-Time of Flight Mass Spectrometry (MALDI-TOF MS) Alone versus MALDI-TOF MS Combined with Real-Time Antimicrobial Stewardship Interventions on Time to Optimal Antimicrobial Therapy in Patients with Positive Blood Cultures. Munson E, editor. J Clin Microbiol. 2017;55:1437-45

Ready to submit your research? Choose BMC and benefit from:

- fast, convenient online submission

- thorough peer review by experienced researchers in your field

- rapid publication on acceptance

- support for research data, including large and complex data types

- gold Open Access which fosters wider collaboration and increased citations

- maximum visibility for your research: over $100 \mathrm{M}$ website views per year

At $\mathrm{BMC}$, research is always in progress.

Learn more biomedcentral.com/submissions 